\title{
COMMENTARY
}

\section{Pharmacists Leading the Way to Precision Medicine: Updates to the Core Pharmacist Competencies in Genomics}

\author{
Roseann S. Gammal, PharmD, ${ }^{\mathrm{a}, \mathrm{b}}$ Yee Ming Lee, PharmD, ${ }^{\mathrm{b}, \mathrm{c}}$ Natasha J. Petry, PharmD, MPH, ${ }^{\text {b,d }}$ Otito \\ Iwuchukwu, RPh, PhD, ${ }^{\text {be }}$ James M. Hoffman, PharmD, MS, ${ }^{\text {b,f }}$ David F. Kisor, PharmD, ${ }^{\text {b,g }}$ Philip E. Empey, \\ PharmD, $\mathrm{PhD}^{\mathrm{b}, \mathrm{h}}$ \\ ${ }^{a}$ Massachusetts College of Pharmacy and Health Sciences, Boston, Massachusetts \\ ${ }^{\mathrm{b}}$ American Association of Colleges of Pharmacy Pharmacogenomics Special Interest Group, Arlington, Virginia \\ ${ }^{c}$ University of Colorado, Skaggs School of Pharmacy and Pharmaceutical Sciences, Aurora, Colorado \\ ${ }^{\mathrm{d}}$ North Dakota State University, School of Pharmacy, Fargo, North Dakota \\ ${ }^{\mathrm{e}}$ Farleigh Dickinson University, School of Pharmacy, Florham Park, New Jersey \\ ${ }^{\mathrm{f}}$ St. Jude Children's Research Hospital, Memphis, Tennessee \\ ${ }^{\mathrm{g}}$ Manchester University, North Manchester, Indiana \\ ${ }^{\text {h }}$ University of Pittsburgh School of Pharmacy, Pittsburgh, Pennsylvania
}

Corresponding Author: Roseann S. Gammal, Massachusetts College of Pharmacy and Health Sciences, 179 Longwood Ave, Boston, MA 02115. Tel: 617-735-1052. Email: roseann.gammal@mcphs.edu

Submitted March 9, 2021; accepted June 23, 2021; ePublished July 2021

Genomics is increasingly becoming an important part of health care, and pharmacists are well-positioned to be practicebased leaders in pharmacogenomics and precision medicine. Competencies available through the Genetics/Genomics Competency Center provide a framework for pharmacogenomics instruction in both pharmacy school curricula and continuing education programs. Given the significant advancements in pharmacogenomics over the past decade, the 20192020 American Association of Colleges of Pharmacy Pharmacogenomics Special Interest Group updated the pharmacist competencies. The process used a systematic approach which included mapping pharmacogenomics-specific competencies to the Entrustable Professional Activities for pharmacists and seeking consensus from key stakeholders. The final result is an expansion to 30 competencies that reflect the contemporary roles pharmacists play in the application of pharmacogenomics in clinical practice. When implemented into curricula, these competencies will ensure that learners are "practice-ready" to integrate pharmacogenomics into patient care. Additional post-graduate training is needed for advanced roles in pharmacogenomics implementation, education, and research.

Keywords: pharmacogenomics, pharmacogenetics, pharmacy, education, competencies

\section{INTRODUCTION}

Genomics is increasingly becoming an important part of health care, and pharmacists across practice settings have important roles to play. The recently published National Human Genome Research Institute (NHGRI) strategic vision for "improving human health at The Forefront of Genomics" highlights how pharmacists and pharmacy systems can support the implementation of genomics into clinical care. ${ }^{1}$ With extensive education and training in pharmacology, including pharmacokinetics and pharmacodynamics, and the optimal use of medications, pharmacists are the health care professionals best suited to lead efforts to integrate pharmacogenomics into clinical practice, an idea uniformly supported by major professional pharmacy organizations. ${ }^{2-4}$

Pharmacogenomics is a rapidly evolving field, and the profession of pharmacy must advance with it to meet the needs of today's patients. One of the many barriers to deploying pharmacogenomics more broadly in the clinic is the lack of adequate education and training for health care professionals, including pharmacists. ${ }^{5}$ The 2016-17 American

Association of Colleges of Pharmacy (AACP) Argus Commission comprised of the past five AACP presidents recognized the educational needs to support the vision of pharmacists as practice-based leaders in the field, stating that "the most significant threat to pharmacy will be a failure to enrich our curricula and post-graduate education with the adequate intensity of attention to the expanding field of pharmacogenomics..." Although the 2016 Accreditation Standards and Key Elements for the Professional Program in Pharmacy Leading to the Doctor of Pharmacy Degree includes "pharmacogenomics/genetics" as foundational content in pharmacy education and "pharmacogenomic considerations" as a key part of evidence-based clinical decision making, further granularity in characterizing how pharmacists can integrate pharmacogenomics into the Pharmacists' Patient Care Process (PPCP) across the continuum of care is needed. ${ }^{7}$ 
Established competencies provide a standardized framework for pharmacogenomics instruction, helping educators to design learning objectives and prioritize content delivery. In 2012, the AACP Pharmacogenomics Special Interest Group (SIG) led the effort to update the original 2002 pharmacist competencies in genomics, including

pharmacogenomics. ${ }^{8,9}$ The competencies were published in 2016 and disseminated through the NHGRI Genetics/Genomics Competency Center (G2C2) website alongside the genomics-related competency statements for physicians, physician assistants, nurses, and genetic counselors. ${ }^{10}$

Since the latest pharmacist competencies in genomics were published, the profession has seen significant progress in the field, including the development of novel pharmacist-led clinical pharmacogenomics services, United States Food and Drug Administration approval of direct-to-consumer pharmacogenomic testing, and expansion of evidence-based pharmacogenomics clinical practice guidelines. ${ }^{11-14}$ The pharmacy academy has also been introduced to the concept of "Entrustable Professional Activities" (EPAs) - discrete, essential activities that all pharmacy graduates are expected to perform without direct supervision. ${ }^{15}$ As newer areas of practice emerge (eg, clinical pharmacogenomics), mapping the associated core competencies to the EPAs will help define the pharmacist's role in those areas and provide a standard framework for pharmacy education. To support the vital role of pharmacists in advancing pharmacogenomics-based care, the 2019-2020 AACP Pharmacogenomics SIG leadership launched the effort to update the pharmacist competencies in genomics to reflect the needs of contemporary pharmacy practice and map them to the EPAs.

\section{METHODS}

\section{Process to Evaluate and Revise Existing Pharmacist Competencies in Genomics}

The expert group leading the effort to update pharmacist competencies in genomics included seven experienced pharmacogenomics educators and implementers who are current leaders in the AACP Pharmacogenomics SIG or pharmacogenomics experts who authored the previous competencies. The process was conducted in 2020 and included 1) garnering support from key stakeholders, including representatives from NHGRI and AACP; 2) critically analyzing the existing genomics-related competencies for pharmacists and other health care providers; 3 ) developing a revised framework for pharmacist competencies in genomics; 4) conducting a gap analysis of pharmacogenomics-specific competencies relative to the EPAs; 5) systematically reviewing, editing, and adding competency statements to achieve expert consensus; 6) seeking input and approval of the revised statements from leaders/content experts of continuing education programs offered by national pharmacy organizations; and 7) seeking input and approval of the revised statements from the AACP Pharmacogenomics SIG membership. Revising the competency statements was an iterative process that occurred over several months to achieve consensus.

The AACP Executive Vice President and CEO, as well as leadership of the NHGRI Inter-Society Coordinating Committee for Practitioner Education in Genomics, expressed strong support for the project from the beginning. The expert working group first reviewed and compared genomics competencies across professions with a particular focus on pharmacogenomics-related competencies. All experts agreed that the EPAs provided a logical framework from which to build the pharmacogenomics-related competency statements for pharmacists. From there, the prior competencies were mapped back to specific EPAs. Foundational genetics concepts emerged as its own category to capture relevant, but nonpharmacogenomics-related, competencies. Based on the gaps identified through the EPA mapping exercise, additional genomics competencies were created, discussed, and modified iteratively by the expert group through regular meetings occurring over a six-month period. Thus, the EPA framework was a major driver for the final number of competencies. To ensure broader stakeholder input, the draft competencies were disseminated to the AACP Pharmacogenomics SIG membership through the AACP Connect discussion board with an open comment period. The feedback received was used to further refine the competency statements. All members of the expert working group agreed with the final version.

\section{The Updated Pharmacist Competencies in Genomics}

The previous 15 genomics competency statements were originally divided into four categories: Basic Genetic Concepts ( $n=4)$, Genetics and Disease $(n=3)$, Pharmacogenetics/ Pharmacogenomics $(n=3)$, and Ethical, Legal, and Social Implications (ELSI) $(n=5)$. The new competencies (Table 1) are organized into just two categories: Foundational Genetics Concepts $(n=6)$ and Clinical Pharmacogenomics $(n=24)$, with the latter mapped to the established EPAs for pharmacy practice (Table 2). ${ }^{15}$ Competencies specific to the retired categories of Genetics and Disease and ELSI were reviewed, modified, and integrated into the two new sections as appropriate. The final result is an expansion to 30 total competency statements that are more specific to the contemporary roles pharmacists play in the application of genomic medicine, particularly pharmacogenomics, in clinical practice.

The Foundational Genetics Concepts competencies encompass basic genetics principles/nomenclature, the genetics of disease, genetic testing technologies, and legal protections against genetic discrimination. Although pharmacogenomics is the subset of genomic medicine most within the pharmacist's purview, pharmacists must also have 
a working knowledge of core genetics concepts to understand the broader context of how pharmacogenomics intersects with other areas of medical genetics. This idea has persisted since the development of the original 2002 competencies. $^{8}$

A key difference between the most recent competencies and the new ones presented herein is the organization and depth of the pharmacogenomics-related statements. Previously, pharmacogenomics-specific competencies were located in multiple sections; now, they are grouped together in a single section. The rationale for this change is multifold. First, organizing comprehensive competency statements in clinical pharmacogenomics for pharmacists will help further delineate the pharmacist's role as a practice-based leader in the field. The genomics-related competencies for most nonpharmacist health care providers (eg, physician assistants, nurses, and genetic counselors) do not include specific statements pertaining to pharmacogenomics; however, the physician competencies include some general pharmacogenomics-focused statements. ${ }^{10}$ Further, a major change included integrating the previous pharmacogenomics competencies into the existing EPA framework and using the established EPA domains and core statements to address any gaps in pharmacogenomics-specific competencies. This resulted in an expansion of pharmacogenomics-specific competency statements from three to 24. There is only one EPA without a corresponding pharmacogenomics competency ("ensure that patients have been immunized against vaccine-preventable diseases"); all others have one or more corresponding competency statements associated with it. The additional specificity of the new pharmacogenomics competencies provides the detail needed to guide educational initiatives and can be directly used as specific learning objectives across pharmacy curricula. Relevant ELSI topics were weaved throughout, as the experts agreed that these issues should reflect an integrative process of care rather than be viewed as separate issues. These include legal protections against discrimination based on genetic test results (Table 1, FG-6), disease implications of pharmacogenomic test results (Table 1, CP-5), cost/reimbursement considerations (Table 1, CP-11), benefits and limitations of testing (Table 1, CP-19), and a culturally-sensitive approach to counseling patients about their results (Table 1, CP-20). In general, ELSI considerations for pharmacogenomics tend not to be as extensive as those pertaining to genetic testing for disease risk. ${ }^{16}$

\section{Pharmacists as Practice-Based Leaders in Clinical Pharmacogenomics}

As the medication experts, pharmacists have a fundamental responsibility to understand the contributions of all factors that influence medication response, including genetics. Just as pharmacokinetics was once a specialty area of pharmacy practice and is now an expected area of competency for all pharmacists, pharmacogenomics must also make the same transition as the profession evolves. These competencies provide a blueprint for integrating and expanding genomics/pharmacogenomics educational content in PharmD program curricula as well as for continuing education programs geared towards practicing pharmacists.

Pharmacogenomics is relevant for all aspects of the PPCP, from collecting pharmacogenomic data to following up and monitoring a pharmacogenomics-guided care plan. From a patient safety perspective, pharmacogenomics is an important tool that pharmacists can leverage to decrease the risk of, or outright prevent, serious adverse drug reactions. With increasing access to pharmacogenomic testing (eg, via expanding clinical pharmacogenomics services, large-scale research programs such the All of Us research program, and direct-to-consumer genetic tests), pharmacists must be ready to meet the needs of patients and other health care providers with respect to pharmacogenomic test interpretation and application to practice as part of comprehensive medication management. Pharmacists should be the primary health care provider that others turn to for guidance on recommending pharmacogenomic testing to aid in drug selection and dosing; designing medication regimens based on pharmacogenomics and other patient-specific data; and educating patients and clinicians about pharmacogenomics principles and the clinical implications of test results.

Just as the core EPAs provide a clear and comprehensive description of a pharmacist's work, the new pharmacogenomics competencies that map back to those core EPAs illustrate the specific skills that all pharmacists need to possess to provide pharmacogenomics-based care. What it means to be a "practice-ready" pharmacist in the era of genomic medicine must include the interpretation and application of pharmacogenomic test results to patient care. In addition, the mapping process establishes clinical pharmacogenomics as integral to the pharmacist's work, which makes it distinct from other health care professionals' roles. While pharmacists are the logical leaders in pharmacogenomics, other health care professionals also support the clinical implementation of pharmacogenomics. For example, collaborations between pharmacists and genetic counselors have been proposed to communicate the benefits and limitations of genetic testing, familial implications of genetic test results, and the clinical significance of secondary (eg, disease-related) findings, while the pharmacist provides the medication-related knowledge and understanding of how genetics may be integrated with other clinical factors to inform pharmacotherapy decision-making. ${ }^{17}$

Despite the call for increased pharmacogenomics education and training for all pharmacists, there is still a need for some pharmacists to specialize in pharmacogenomics to lead implementation, education, and research efforts. This may require additional residency, fellowship, or graduate school training to achieve advanced competencies. ${ }^{18,19}$ Furthermore, certain specialty areas of practice with advanced applications of genomics (eg, oncology) may also 
necessitate additional competencies that are beyond the scope of this project. The competencies presented in this report were written to ensure a baseline level of genomics/pharmacogenomics literacy for all pharmacists to provide solid foundation of knowledge and skills for contemporary clinical practice. Integrating these new competencies into curricula will require pharmacogenomics-trained faculty to provide both didactic and experiential learning opportunities for students. ${ }^{5,20}$ The updated competencies are disseminated to pharmacy educators through AACP and to the general public via the $\mathrm{G} 2 \mathrm{C} 2$ website. $^{10}$

\section{CONCLUSION}

Clearly defined pharmacist competencies in genomics that are refined as genomic medicine matures are essential to advance pharmacy education and practice in pharmacogenomics and precision medicine. Pharmacists are the health care professionals best suited to lead collaborative, interprofessional teams in the provision of pharmacogenomics-based care across practice settings and therapeutic areas. The competencies described in this report serve as a blueprint for clinical pharmacogenomics instruction as part of pharmacy school curricula and continuing education programs for practicing pharmacists. When implemented into pharmacy school curricula, these competencies will ensure that graduates are "practice-ready" to integrate pharmacogenomics into patient care. Additional post-graduate training is needed for advanced roles in pharmacogenomics implementation, education, and research.

\section{ACKNOWLEDGMENTS}

The authors would like to thank the American Association of Colleges of Pharmacy (AACP) leadership for their support of this project; the National Human Genome Research Institute for sponsoring the Genetics/Genomics Competency Center website and disseminating the updated pharmacist competencies in genomics on that platform; and members of the AACP Pharmacogenomics Special Interest Group for their input and support.

\section{REFERENCES}

1. Green ED, Gunter C, Biesecker LG, et al. Strategic vision for improving human health at The Forefront of Genomics. Nature. 2020;586(7831):683-692.

2. ASHP statement on the pharmacist's role in clinical pharmacogenomics. Am J Health Syst Pharm. 2015;72(7):579-581.

3. Actions of the 2018 American Pharmacists Association House of Delegates. J Am Pharm Assoc. 2018;58:355365.

4. Hicks JK, Aquilante CL, Dunnenberger HM, et al. Precision Pharmacotherapy: Integrating Pharmacogenomics into Clinical Pharmacy Practice. J Am Coll Clin Pharm. 2019;2(3):303-313.

5. Weitzel KW, Aquilante CL, Johnson S, Kisor DF, Empey PE. Educational strategies to enable expansion of pharmacogenomics-based care. Am J Health Syst Pharm. 2016;73(23):1986-1998.

6. Crabtree B, Bootman JL, Boyle CJ, Chase P, Piascik P, Maine LL. Aligning the AACP Strategic Engagement Agenda with Key Federal Priorities in Health: Report of the 2016-17 Argus Commission. Am J Pharm Educ. 2017;81(8):S15.

7. Accreditation Council for Pharmacy Education. Accreditation Standards and Key Elements for the Professional Program in Pharmacy Leading to the Doctor of Pharmacy Degree ("Standards 2016"). Published February 2015. https://www.acpe-accredit.org/pdf/Standards2016FINAL.pdf. Accessed June 21, 2021.

8. Johnson JA, Bootman JL, Evans WE, et al. Pharmacogenomics: A Scientific Revolution in Pharmaceutical Sciences and Pharmacy Practice. Report of the 2001-2002 Academic Affairs Committee. Am J Pharm Ed. 2002;66:12s-15s.

9. Roederer MW, Kuo GM, Kisor DF, et al. Pharmacogenomics competencies in pharmacy practice: A blueprint for change. J Am Pharm Assoc (2003). 2017;57(1):120-125.

10. Genetics/Genomics Competency Center. 2020; https://www.genomicseducation.net/. Accessed June 21, 2021.

11. Dunnenberger HM, Biszewski M, Bell GC, et al. Implementation of a multidisciplinary pharmacogenomics clinic in a community health system. Am J Health Syst Pharm. 2016;73(23):1956-1966.

12. Crews KR, Cross SJ, McCormick JN, et al. Development and implementation of a pharmacist-managed clinical pharmacogenetics service. Am J Health Syst Pharm. 2011;68(2):143-150.

13. Gammal RS, Mayes J, Caudle KE. Ready or not, here it comes: Direct-to-consumer pharmacogenomic testing and its implications for community pharmacists. J Am Pharm Assoc (2003). 2019;59(5):646-650.

14. Relling MV, Klein TE, Gammal RS, Whirl-Carrillo M, Hoffman JM, Caudle KE. The Clinical Pharmacogenetics Implementation Consortium: 10 Years Later. Clin Pharmacol Ther. 2020;107(1):171-175. 
15. Haines ST, Pittenger AL, Stolte SK, et al. Core Entrustable Professional Activities for New Pharmacy Graduates. Am J Pharm Educ. 2017;81(1):S2.

16. Johnson JA. Pharmacogenetics in clinical practice: how far have we come and where are we going? Pharmacogenomics. 2013;14(7):835-843.

17. Zierhut HA, Campbell CA, Mitchell AG, Lemke AA, Mills R, Bishop JR. Collaborative Counseling Considerations for Pharmacogenomic Tests. Pharmacotherapy. 2017;37(9):990-999.

18. Haidar CE, Hoffman JM, Gammal RS, Relling MV, Crews KR. Development of a postgraduate year 2 pharmacy residency in clinical pharmacogenetics. Am J Health Syst Pharm. 2017;74(6):409-415.

19. Required Competency Areas, Goals, and Objectives for Postgraduate Year Two (PGY2) Clinical Pharmacogenomics Pharmacy Residencies. https://www.ashp.org/-/media/assets/professionaldevelopment/residencies/docs/pgy2-clinical-pharmacogenomicscago.ashx?la=en\&hash=495588AB3FB0C845C8714E93E5D6D685373DB564. Accessed June 21, 2021.

20. Gammal RS, Nguyen J, Audi E, et al. Advanced Pharmacy Practice Experiences in Pharmacogenomics Offered by US Pharmacy Programs. Am J Pharm Educ. 2020;84(12):8031. 
Table 1. Core pharmacist competencies in genomics

Foundational Genetics Concepts (FG)

FG-1 Explain basic genetics concepts using appropriate nomenclature.

FG-2 Recognize the combined impact of genetic, behavioral, social, and environmental factors in the manifestation of disease and drug response.

FG-3 Identify drug- and disease-associated genetic variations that facilitate development of prevention, diagnostic, and treatment strategies.

FG-4 Differentiate between the clinical diagnosis of disease informed by genetics and the identification of genetic predisposition to disease.

FG-5 Assess differences in genetic testing technologies, including sequencing and genotyping.

FG-6 Recognize the legal protections against discrimination based on genetic test results.

Clinical Pharmacogenomics (CP)

EPA Domain: Patient Care Provider

CP-1 Identify pharmacogenomic test results that are relevant to a patient's care.

CP-2 Interpret pharmacogenomic test results, including translating genotype to phenotype to drug therapy recommendation.

CP-3 Determine the impact of genetic variation on pharmacokinetics and/or pharmacodynamics.

CP-4 Identify medication-related problems that may be related to genetic variability, even when a pharmacogenomic test has not been done.

CP-5 Recognize disease implications of pharmacogenomic test results and refer the patient to a genetics-trained healthcare provider when necessary.

CP-6 Use family history to assess predisposition to disease and drug response.

CP-7 Assess the quality and source of existing pharmacogenomic test results.

CP-8 Distinguish between actionable and non-actionable pharmacogenomic test results using high-quality, evidence-based pharmacogenomics databases and clinical guidelines.

CP-9 Integrate pharmacogenomic test results with other clinical variables to optimize medication therapy.

CP-10 Recommend pharmacogenomic testing when appropriate.

CP-11 Consider the cost, cost-effectiveness, and reimbursement issues relevant to pharmacogenomic tests and services.

CP-12 Implement a pharmacogenomics-guided care plan in collaboration with the patient, caregivers, and other health professionals.

CP-13 Document pharmacogenomic test results in the electronic health record.

CP-14 Follow-up and monitor a pharmacogenomics-guided care plan.

EPA Domain: Interprofessional Team Member

CP-15 Collaborate as a member of an interprofessional team as the pharmacogenomics expert.

\section{EPA Domain: Population Health Promoter}

CP-16 Identify patient populations that may be most likely to benefit from pharmacogenomic testing.

CP-17 Identify genetic variations that predispose patients to adverse drug reactions and modify therapy accordingly to mitigate the risk.

CP-18 Recognize the differences in pharmacogenomic allele frequencies among ancestry groups to guide appropriate test selection and maximize the appropriate use of medications in a population.

\section{EPA Domain: Information Master}

CP-19 Educate patients and professional colleagues on the benefits and limitations of pharmacogenomics to optimize drug therapy.

CP-20 Use a culturally-sensitive approach that considers potential ethical concerns when counseling patients about pharmacogenomic test results.

CP-21 Use evidence-based resources and pharmacogenomics information to advance patient care.

EPA Domain: Practice Manager

CP-22 Oversee pharmacy operations that integrate pharmacogenomics for an assigned work shift. 

Table 2. Pharmacist competencies in clinical pharmacogenomics mapped to the core entrustable professional activities (EPAs) for pharmacy practice

\begin{tabular}{ll}
\hline EPA Domain & EPA Core Statements \\
\hline Patient Care Provider & $\begin{array}{l}\text { Collect information to identify a patient's } \\
\text { medication-related problems and health-related } \\
\text { needs. }\end{array}$ \\
& $\begin{array}{l}\text { Analyze information to determine the effects of } \\
\text { medication therapy, identify medication-related }\end{array}$
\end{tabular}
problems, and prioritize health-related needs.

\section{Clinical Pharmacogenomics Competencies}

1. Identify pharmacogenomic test results that are relevant to a patient's care.

2. Interpret pharmacogenomic test results, including translating genotype to phenotype to drug therapy recommendation.

3. Determine the impact of genetic variation on pharmacokinetics and/or pharmacodynamics.

4. Identify medication-related problems that may be related to genetic variability, even when a pharmacogenomic test has not been done.

5. Recognize disease implications of pharmacogenomic test results and refer the patient to a genetics-trained healthcare provider when necessary.

6. Use family history to assess predisposition to disease and drug response.

7. Assess the quality and source of existing pharmacogenomic test results.

8. Distinguish between actionable and non-actionable pharmacogenomic test results using high-quality, evidence-based pharmacogenomics databases and clinical guidelines.

Establish patient-centered goals and create a care plan for a patient in collaboration with the patient, caregiver(s), and other health professionals that is evidence-based and cost-effective.

9. Integrate pharmacogenomic test results with other clinical variables to optimize medication therapy.

10. Recommend pharmacogenomic testing when appropriate.

11. Consider the cost, cost-effectiveness, and reimbursement issues relevant to pharmacogenomic tests and services.

Implement a care plan in collaboration with the patient, caregivers, and other health professionals.

12. Implement a pharmacogenomics-guided care plan in collaboration with the patient, caregivers, and other health professionals.

13. Document pharmacogenomic test results in the electronic health record. 
Follow-up and monitor a care plan.

Collaborate as a member of an interprofessional team.

Interprofessiona

Team Member

Population Health

Promoter

Identify patients at risk for prevalent diseases in a population.

Minimize adverse drug events and medication errors.

Maximize the appropriate use of medications in a population

Ensure that patients have been immunized against vaccine-preventable diseases.

Information Master

Educate patients and professional colleagues regarding the appropriate use of medications.

Use evidence-based information to advance patient care.

Practice Manager

Oversee the pharmacy operations for an assigned work shift.

Fulfill a medication order.

Create a written plan for continuous professional development.
14. Follow-up and monitor a pharmacogenomics-guided care plan.

15. Collaborate as a member of an interprofessional team as the pharmacogenomics expert.

16. Identify patient populations that may be most likely to benefit from pharmacogenomic testing.

17. Identify genetic variants that predispose patients to adverse drug reactions and modify therapy accordingly to mitigate the risk.

18. Recognize the differences in pharmacogenomic allele frequencies among ancestry groups to guide appropriate test selection and maximize the appropriate use of medications in a population.

19. Educate patients and professional colleagues on the benefits and limitations of pharmacogenomics to optimize drug therapy.

20. Use a culturally-sensitive approach that considers potential ethical concerns when counseling patients about pharmacogenomic test results.

21. Use evidence-based resources and pharmacogenomics information to advance patient care.

22. Oversee pharmacy operations that integrate pharmacogenomics for an assigned work shift.

23. Fulfill a medication order considering the clinical implications of pharmacogenomics.

24. Create a written plan for continuous professional development in clinical pharmacogenomics. 